Armin Pfabl-Tranghber

\title{
Islamismus - der neue Extremismus, Faschismus, Fundamentalismus und Totalitarismus? Eine Erörterung zu Angemessenheit und Erklärungskraft der Zuordnungen
}

\section{Einleitung und Fragestellung}

Seit den Anschlägen vom 11. September 2001 steht der Islamismus verstärkt im Fokus des öffentlichen und wissenschaftlichen Interesses. Dabei lassen sich zwei Fehlwahrnehmungen ausmachen: Zum einen handelt es sich um kein neues Phänomen, zum anderen gibt es nicht nur eine terroristische Variante. Das Ausmaß der Anschläge und ihre weltpolitischen Folgen forcierten offenbar eine solche Sicht der Dinge. Hierdurch erklärt sich auch, warum in Stellungnahmen von Journalisten und Politikern, $\mathrm{Pu}$ blizisten und Wissenschaftlern Begriffe, Kategorien und Zuschreibungen aus anderen inhaltlichen Kontexten auftauchen. Um den Islamismus inhaltlich zuzuordnen, bedienen sich viele Betrachter Ansätzen und Bezeichnungen, welche aus ganz anderen politischen Kontexten stammen, aber offensichtlich über Gemeinsamkeiten mit dem Islamismus verfügen. Dabei stellt man auf bestimmte Auffälligkeiten solcher politischer Bestrebungen ab, begründet aber nur selten die Angemessenheit der Begriffswahl.

Es kursieren etwa die Bezeichnungen »Extremismus « und »Faschismus«, »Fundamentalismus « und »Totalitarismus«: So sprechen Historiker und Islamwissenschaftler, Politikwissenschaftler und Soziologen vom »dritten Extremismus « (Miroslav Mares) « ${ }^{1}$ oder »religiösem Extremismus «(Steffen Kailitz) ${ }^{2}$, vom » Klerikalfaschismus « (Walter Laqueur) ${ }^{3}$ oder einer »Form des Rechtsextremismus« (Ursula Birsl u. a.) $)^{4}$, vom »islamischen Fundamentalismus « (Khadija Katja Wöhler-Khalfallah) ${ }^{5}$ oder »religiösen Fundamentalismus« (Klaus Kienzler) ${ }^{6}$, vom »dritten Totalitarismus« (Yeuda

1 Vgl. Miroslav Mares, Der dritte Extremismus. Islamistische Bewegung aus der Sicht der Politikforschung, in: Freiheit und Recht, Nr. 2/Juni 2007, S. 5-7.

2 Vgl. Steffen Kailitz, Politischer Extremismus in der Bundesrepublik Deutschland. Eine Einführung, Wiesbaden 20ß04, S. 164-183.

3 Vgl. Walter Laqueur, Faschismus. Gestern - heute - morgen, Berlin 1997, S. 225-259.

4 Vgl. Ursula Birsl/Ersen Bucak/Can Zeyrek, Religiöser Fundamentalismus oder politischer Rechtsextremismus? Islamistische Organisationen und Aktivitäten in der Bundesrepublik, in: Blätter für deutsche und internationale Politik 47 Jg., Nr. 6 vom Juni, 2002, S. 720-727.

5 Vgl. Khadia Katja Wöhler-Khalfallah, Der islamische Fundamentalismus, der Islam und die Demokratie. Algerien und Tunesien: das Scheitern postkolonialer »Entwicklungsmodelle « und das Streben nach einem ethischen Leitfaden für Politik und Gesellschaft, Wiesbaden 2006, S. 24-33.

6 Vgl. Klaus Kienzler, Der religiöse Fundamentalismus. Christentum - Judentum - Islam, München 1996, S. 72-94. 
Bauer $)^{7}$ oder »neuen Totalitarismus « (Bassam Tibi) ${ }^{8}$. Die jeweils damit verbundenen Ansätze und Begriffe, Konzepte und Theorien entstammen aber der inhaltlichen Auseinandersetzung mit ganz anderen politischen und religiösen Bestrebungen: dem Links- und Rechtsextremismus, dem Faschismus und Nationalsozialismus, den USEvangelikalen und dem orthodoxen Islam sowie dem Hitler- und Stalin-Regime.

Wie angemessen und erkenntnisfördernd ist es nun, die erwähnten begrifflichen Zuordnungen auf den Islamismus anzuwenden? Dies stellt die Hauptfragestellung der vorliegenden Erörterung dar. Sie soll wie folgt angegangen werden: Zunächst bedarf es einer Definition von Ideologie und Handlungsstilen des zu analysierenden Phänomens in Gestalt des Islamismus (2). Danach werden die einzelnen Begründungen für die Begriffsverwendung in der bisherigen Debatte referiert und mit dem Verständnis der genannten Konzepte in der Forschung konfrontiert. Dem folgt eine inhaltliche Auseinandersetzung mit den damit verbundenen Argumenten und eine bilanzierende Einschätzung zur jeweiligen Zuordnung. Im Einzelnen geht es um »Extremismus « (3.), »Faschismus« (4.), »Fundamentalismus « (5.) und »Totalitarismus « (6.), konkret bezogen auf die formale und inhaltliche Angemessenheit und den analytischen und theoretischen Nutzen für die Bewertung und den Umgang mit dem zu örternden Phänomen.

\section{Islamismus - Definition und Handlungsstil}

»Islamismus « steht für eine Sammelbezeichnung unterschiedlicher Strömungen einer politischen Bewegung 9 , die ihren ideologischen Ursprung in inner-islamischen Reformbestrebungen in der zweiten Hälfte des 19. Jahrhunderts ${ }^{10}$ und ihre organisatorischen Wurzeln in der 1928 in Ägypten gegründeten »Muslimbruderschaft « ${ }^{11}$ hat. Allen späteren Strömungen ist die Absicht eigen, den Islam nicht nur zur verbindlichen Leitlinie für das individuelle, sondern auch für das gesellschaftliche Leben $\mathrm{zu}$ machen. Dies bedeutet notwendigerweise die Aufhebung einer Trennung von Religion und Staat als Ausdruck der Säkularisierung und die institutionelle Verankerung der religiösen Grundlagen im Sinne eines islamischen Staates. Damit einher geht die Ablehnung der Prinzipien von Individualität, Menschenrechten, Pluralismus, Säkularisierung und Volkssouveränität. ${ }^{12}$ Insofern nimmt der Islamismus

7 Vgl. Yehuda Bauer, Der dritte Totalitarismus. Radikale Islamisten kämpfen um die Weltherrschaft. Das haben sie mit Hitler und Stalin gemein, in: Die Zeit, Nr. 32 vom 31. Juli 2003, S. 11.

8 Vgl. Bassam Tibi, Der neue Totalitarismus. »Heiliger Krieg« und westliche Sicherheit, Darmstadt 2004, S. 19.

9 Vgl. Gilles Kepel, Das Schwarzbuch des Dschihad. Aufstieg und Niedergang des Islamismus, München 2002.

10 Vgl. Peter Heine, Terror in Allahs Namen. Extremistische Kräfte im Islam, Freiburg 2001, S. 86-116.

11 Vgl. Richard P. Mitchell, The Society of the Muslim Brothers, New York 1993.

12 Vgl. Olaf Farschid; Islam als System: Grundzüge islamistischer Ideologie, in: Senatsverwaltung für Inneres (Hrsg.), Islamismus. Diskussion eines vielschichtigen Phänomens, Berlin 2005, S. 14-26. 
eine Frontstellung gegen das normative Selbstverständnis offener Gesellschaften und demokratischer Verfassungsstaaten ein.

Bei allen darauf bezogenen ideologischen Gemeinsamkeiten lassen sich hinsichtlich der politischen Artikulations- und Handlungsweise Unterschiede ausmachen. Entgegen einem weit verbreiteten Eindruck sind weder alle Islamisten gewalttätig noch terroristisch ausgerichtet. Statt einer solchen Pauschalisierung empfiehlt sich eine Unterscheidung von gewaltbereiten und reformorientierten Varianten ${ }^{13}$, die sich wiederum in zwei weitere Subgruppen differenzieren lassen. Zu dem letztgenannten Typus gehört eine kulturelle Form, die über das Engagement im Alltagsleben Akzeptanz für ihre Auffassungen mobilisieren will, und eine politische Variante, die über Parteipolitik große Unterstützung für sich unter Wählern anstrebt. Die gewaltbereite Form kann danach unterschieden werden, ob ihre Anhänger regional oder transnational ausgerichtet sind. Als Kriterium für die Unterscheidung gilt hierbei der Zielort solcher Aktivitäten, die sich auf das Herkunftsland beschränken oder weit darüber hinausgehen können. ${ }^{14}$

Die vorgenommene Definition der Ideologie und Unterscheidung der Handlungsstile versteht sich als abstrakt und idealtypisch, kann man doch auch Gemeinsamkeiten mit der Religion selbst oder Mischformen mit anderen Vorgehensweisen ausmachen. Hinsichtlich des ersten Gesichtspunktes gilt es darauf hinzuweisen, dass der Islam als Religion der Muslime vom Islamismus als politischer Bestrebung unterscheidbar ist, sich aber in Basis und Geschichte dieses Glaubens durchaus Anknüpfungspunkte für eine solche Ideologie finden. ${ }^{15}$ Dies gilt insbesondere für das Verständnis des Islam als Gesellschafts- und Rechtsordnung. Hinsichtlich des zweiten Gesichtspunktes lassen sich einzelne Organisation eindeutig einem bestimmten Handlungsstil zuordnen wie etwa die »Islamische Gemeinschaft Milli Görüs «16 in Deutschland zur kulturellen Form der reformorientierten Variante, während eine Organisation wie die »Hamas «17 sowohl auf diesem Feld wie der Gewalthandlung und Parteipolitik aktiv ist.

13 Bassam Tibi unterscheidet in diesem Sinne einen »jihadistischen « und »institutionellen Islamismus «, vgl. Tibi, Der neue Totalitarismus (Anm. 13), S. 14f. und 75.

14 Insofern widerspricht diese Differenzierung auch nicht der Auffassung von Guido Steinberg, wonach man bei dem Blick auf den transnationalen Charakter islamistischer Terror-Netzwerke nicht die lokalen Bestimmungs- und Zielfaktoren ignorieren dürfe. Vgl. Guido Steinberg, Der nahe und der ferne Feind. Die Netzwerke des islamistischen Terrorismus, München 2005, S. 9f. und 236f.

15 Vgl. Armin Pfahl-Traughber, Die Islamismuskompatiblität des Islam. Anknüpfungspunkte in Basis und Geschichte der Religion, in: Aufklärung und Kritik, Sonderheft 13/ 2007, Schwerpunkt: Islamismus, S. 62-78.

16 Vgl. Bundesministerium des Innern (Hrsg.), Verfassungsschutzbericht 2006, Berlin 2007, S. 239-251.

17 Vgl. Joseph Croitoru, Hamas. Der islamische Kampf um Palästina, München 2007. 


\section{Angemessenheit der Extremismus-Zuordnung}

\subsection{Extremismus - Begründung und Definition}

Die Subsumtion des Islamismus unter den Extremismusbegriff erfolgte zunächst durch die Verfassungsschutzbehörden. Sie sprachen im Kontext von »sicherheitsgefährdenden und extremistischen Bestrebungen von Ausländern « ab 1983 von »islamischen Extremisten « ${ }^{18}$, ab 1994 von »Islamisten $\ll^{19}$ und formulierten bereits vor den Anschlägen vom 11. September 2001 eine kurze theoretische Zuordnung. Sie stellte darauf ab, dass die eingeforderte islamische Gesellschaftsordnung Grundsätzen der freiheitlichen demokratischen Grundordnung wie dem Gleichheitsgrundsatz oder der Volkssouvänität widerspreche. ${ }^{20}$ Die auf die Phänomenbereiche Linksund Rechtsextremismus fixierte politikwissenschaftliche Extremismusforschung reagierte erst in Folge der erwähnten Anschläge und erweiterte ihr diesbezügliches Verständnis auf den »politisch-religiösen Fundamentalismus«, welcher sich aufgrund seines exklusiven Wahrheitsanspruchs gegen gesellschaftliche Pluralität wende. ${ }^{21}$

Das juristische Verständnis von Extremismus leitet sich aus einer gesetzlichen Grundlage ab. Danach besteht die Aufgabe der Verfassungsschutzbehörden in der Sammlung und Auswertung von Informationen über Bestrebungen, die gegen die freiheitliche demokratische Grundordnung und gegen den Gedanken der Völkerverständigung gerichtet sind..$^{22}$ Gemeint ist damit nicht das Grundgesetz in Gänze, sondern sein normativer Kernbestand. Das verfassungschützerische Verständnis bezieht sich darüber hinaus auf eine politisch aktive Tätigkeit in diesem Sinne und nicht allein auf die Akzeptanz entsprechender Gesinnungen. Daher stehen in der Regel Personengruppen im Zentrum des Interesses einer derartigen Sicherheitsbehörde. Eine genauere inhaltliche Bestimmung von »Extremismus « erfolgt bei dieser juristisch geprägten Definition nicht, beschränkt sie sich doch auf die Definition der von den gemeinten Bestrebungen abgelehnten Prinzipien (freiheitlich demokratische Grundordnung, Gedanke der Völkerverständigung).

Ein solches Verständnis von Extremismus als Abgrenzungsbegriff oder NegativDefintion prägt auch die politikwissenschaftliche Auffassung, die auf die Ablehnung der Minimalbedingungen eines demokratischen Verfassungsstaates abstellt.

18 Vgl. Bundesministerium des Innern (Hrsg.), Verfassungsschutzbericht 1983, Bonn 1984, S. $178 f$.

19 Vgl. Bundesministerium des Innern (Hrsg.), Verfassunsschutzbericht 1994, Bonn 1995, S. $191 \mathrm{f}$.

20 Vgl. Bundesministerium des Innern (Hrsg.), Verfassungsschutzbericht 2000, Berlin Bonn 2001, S. 181.

21 Vgl. Uwe Backes/Eckhard Jesse, Islamismus - Djihadismus - Totalitarismus - Extremismus, in: Uwe Backes/Eckhard Jesse (Hrsg.), Jahrbuch Extremismus \& Demokratie Bd. 14, Baden-Baden 2002, S. 13-26.

22 Vgl. \3 (1) des Bundesverfassungsschutzgesetzes, abgedruckt in: Verfassungsschutzbericht 2006 (Anm. 16), S. 359-377, hier S. 359. 
Dazu gehören das Mehrheitsprinzip verbunden mit einem Minderheitenschutz, die konstitutionelle Verankerung von Bürger- und Menschenrechten, die rechtsstaatliche Verfasstheit, die Verteilung der Gewalten, das konkurrenztheoretische Verständnis von Demokratie, gesellschaftlicher und politischer Pluralismus und das parlamentarische Repräsentativprinzip..$^{23}$ Über diese Negativ-Definition hinaus weist eine Positiv-Definition von Extremismus, die sich auf formale Gemeinsamkeiten der extremistischen Doktrine bei der Ablehnung derartiger Minimalbedingungen bezieht. ${ }^{24}$ Hierzu zählt man offensive und defensive Absolutheitsansprüche, Dogmatismus, Utopismus bzw. kategorischer Utopie-Verzicht, Freund-Feind-Stereotype, Verschwörungstheorien, Fanatismus und Aktivismus. ${ }^{25} 26$

\subsection{Extremismus - Analyse und Kritik}

Für die Einordnung des Islamismus in den politischen Extremismus stellt sich zunächst das Problem, dass er nicht den bekannten Formen des Links- und Rechtsextremismus zugeordnet werden kann. Zwar bestehen formale Gemeinsamkeiten in Form der Absolutheitsansprüche und des Dogmatismus oder der jeweiligen Feindbilder in Gestalt von Israel und den USA. ${ }^{27}$ Daraus lässt sich aber keine inhaltliche Gleichsetzung oder Zuordnung der erwähnten Extremismusphänome vornehmen, existieren doch grundlegende Unterschiede auf der Ebene des ideologischen Selbstverständnisses: Für den Islamismus bildet die besondere Interpretation des Islam den ideologischen Ausgangspunkt, für den Linksextremismus die soziale Ausdehnung des Gleichheitspostulats und für den Rechtsextremismus die Überbetonung der ethnischen Zugehörigkeit. Der Islamismus unterscheidet sich demnach vom Linksextremismus durch seine religiöse Komponente und vom Rechtsextremismus durch sein ethnisches Desinteesse.

Gleichwohl spricht diese Feststellung nicht gegen die Zuordnung des Islamismus zum politischen Extremismus, wofür es lediglich der Benennung einer eigenen Unterkategorie für dieses besondere Phänomen bedürfte. Hier bietet sich die Formu-

23 Vgl. Uwe Backes, Politischer Extremismus in demokratischen Verfassungsstaaten. Eine normative Rahmentheorie, Opladen 1989, S. 87-112.

24 Vgl. Armin Pfahl-Traughber, Politischer Extremismus - was ist das überhaupt? Zur Definition von und Kritik an einem Begriff, in: Bundesamt für Verfassungsschutz (Hrsg.), Bundesamt für Verfassungsschutz. 50 Jahre im Dienst der inneren Sicherheit, Köln 2000, S. 185- 211.

25 Vgl. Backes, Politischer Extremismus in demokratischen Verfassungsstaaten (Anm. 23), S. 298-311.

26 Vgl. in ähnlichem Sinne mit anderen Merkmalen: Armin Pfahl-Traughber, Gemeinsamkeiten im Denken der Feinde einer offenen Gesellschaft. Strukturmerkmale extremistischer Ideologien, in: Martin H. W. Möllers/Robert Chr. van Ooyen (Hrsg.), Jahrbuch Öffentliche Sicherheit 2006/2007, Frankfurt/M. 2007, S. 21-36.

27 Vgl. Armin Pfahl-Traughber, Ideologien des islamistischen, linken und rechten Extremismus in Deutschland. Eine vergleichende Betrachtung, in: Uwe Backes/Eckhard Jesse (Hrsg.), Gefährdungen der Freiheit. Extremistische Ideologien im Vergleich, Göttingen 2006, S. 205-221. 
lierung »religiöser Extremismus « an, worunter politische Bestrebungen gegen die Minimalbedingungen eines demokratischen Verfassungsstaates im angeblichen oder tatsächlichen Namen einer Religion subsummiert werden könnten. Beim Islamismus handelt es sich noch nicht einmal um das einzige derartige Phänomen, könnte man doch ohne weiteres die »Scientology-Organisation $\aleph^{28}$ als gewaltfreie wie die $»$ Aum-Organisation ${ }^{29}$ als gewaltorientierte Form der religiösen Variante des politischen Extremismus ansehen. Entscheidend im Sinne der oben erwähnten NegativDefinition wäre lediglich, dass es sich unabhängig von der ideologischen Ausrichtung um eine politische Bestrebung gegen die Minimalbedingungen des demokratischen Verfassungsstaates handelt.

Dies lässt sich für den Islamismus exemplarisch anhand der Ablehnung der Volksouveränität belegen: Im ideologischen Selbstverständnis von deren Anhängern leitet sich die verbindliche Gestaltung des sozialen Miteinanders in dem angestrebten politischen System nicht von den Menschen, sondern von Gott ab. ${ }^{30} \mathrm{Da}$ er sich aber nicht direkt artikuliert, läuft die Gestaltung einer solchen Gesellschaftsordnung auf die Interpretation seines Willens durch eine elitäre Gruppe hinaus. Damit wird ein tragendes Prinzip moderner Demokratie abgelehnt. Allein von daher kann der Islamismus als Bestandteil eines »dritten Extremismus « ${ }^{31}$ gelten. Darüber hinaus weist dessen Ideologie auch die typischen Strukturmerkmale extremistischer Doktrine auf: exklusiver Erkenntnisanspruch, dogmatischer Absolutheitsanspruch, essentialistisches Deutungsmonopol, holistische Steuerungsabsichten, deterministisches Geschichtsbild, identitäre Gesellschaftskonzeption, dualistischer Rigorismus und fundamentale Verwerfung. ${ }^{32}$

\section{Angemessenheit der Faschismus-Zuordnung}

\subsection{Faschismus - Begründung und Definition}

Die Deutung des Islamismus als »grünen Faschismus « oder »Islam-Faschismus«, die man auch bei bedeutenden Philosophen ${ }^{33}$ und Politikern ${ }^{34}$ ausmachen kann, soll

$28 \mathrm{Vgl}$. Andreas Klump, Neuer politischer Extremismus? Eine politikwissenschaftliche Fallstudie am Beispiel der Scientology-Organisation, Baden-Baden 2003.

29 Vgl. Thomas Gandow, Das Beispiel der AUM Shinri-Kyo (Japen), in: Berndt Georg Thamm, Terrorismus. Ein Handbuch über Täter und Opfer, München 2002, S. 351-380.

30 Vgl. Sayyid Abul A'la Maududi, Islamische Lebensweise, München 1996, S. 62f., wo einer der »Klassiker « islamistischer Ideologie dies in aller Deutlichkeit formuliert.

31 Vgl. Mares, Der dritte Extremismus (Anm. 1).

32 Vgl. Armin Pfahl-Traughber, Islamismus als extremistisches und totalitäres Denken. Strukturmerkmale einer Ideologie der geschlossenen Gesellschaft, in: Aufklärung und Kritik, Sonderheft 13/2002, Schwerpunkt: Islamismus, S. 79-95.

33 Vgl. »Ein Krieg um die Aufklärung «. Der französische Philosoph Bernard-Henry Levy über den Antiamerikanismus europäischer Intellektueller, den Kampf gegen den Terrorismus und die These vom »Ende der Geschichte«, in: Der Spiegel, Nr. 49 vom 3. Dezember 2001, S. 208-212, hier S. 209.

34 Vgl. Katha Pollitt, Falscher Krieg, falsches Wort, in: Die Tageszeitung vom 1. September 2006, S. 11. 
nun erörtert werden. Hierbei lassen sich zwei verschiedene Argumentationsmuster ausmachen: Zum einen verweist man auf die in den 1930er und 1940er Jahren erfolgte historisch-politische Kooperation islamistischer Kräfte mit dem Nationalsozialismus, woraus eine politische Verwandtschaft beider Ideologien und Strömungen abgeleitet wird. Zum anderen stützt sich die Zuordnung auf eine Reihe von formalen Gemeinsamkeiten, die etwa in der Ablehnung von Liberalität und Menschenrechten und der Befürwortung einer elitären Führerschaft, der Unterordnung des Individuums unter das Kollektiv und dem Einsatz von Propaganda und Terror zum Ausdruck komme. ${ }^{35}$ Insbesondere der hier schon enthaltene Gesichtspunkt der Einbettung und Unterordnung des Einzelnen in eine Gemeinschaft gilt als herausragende Gemeinsamkeit. ${ }^{36}$

Über das Faschismusverständnis besteht in der Forschung kein allgemeiner Konsens, bildeten sich doch im Laufe der Zeit die unterschiedlichsten Ansätze heraus. ${ }^{37}$ Die Konfliktlinien der Deutung beziehen sich zum einen auf die Frage, inwieweit der Begriff auch auf Bestrebungen über das Mussolini-Regime in Italien hinaus Anwendung finden kann, und zum anderen auf das Problem, inwieweit das Aufkommen solcher Bewegungen und Systeme mit den Krisen bürgerlicher und kapitalistischer Gesellschaften zusammenhängt. Die im letztgenannten Kontext vertretene Auffassung, welche den Faschismus als eine Art »Agenten des Monopolkapitals« deutet, kann spätestens nach den Forschungen über den Zusammenhang von Faschismus bzw. Nationalsozialismus und Wirtschaft als gescheitert gelten. ${ }^{38}$ Hinsichtlich des erstgenannten Gesichtspunktes besteht die Schwierigkeit insbesondere darin, dass das Hitler-Regime mit der Totalität seiner Herrschaft und der Vernichtungspolitik gegenüber den Juden eine Besonderheit darstellt.

Daraus lässt sich aber nicht notwendigerweise ein Verzicht auf den Faschismusbegriff als Sammelbezeichnung für unterschiedliche politische Bewegungen und Systeme ableiten. Ihnen sind in bestimmten Bereichen bedeutende Spezifika eigen, welche angesichts noch bestehender Unterschiede in anderern Feldern nicht zum Verzicht auf einen gemeinsamen Typusbegriff nötigen. Gerade angesichts der Tatsache, dass es in den 1920er bis 1940er Jahren in zahlreichen europäischen Ländern politische Bewegungen gab, welche sich am deutschen Nationalsozialismus oder italienischen Faschismus orientierten ${ }^{39}$, erlaubt die weitere Verwendung einer solchen Sammelbezeichnung. Als Gemeinsamkeiten gelten auf der ideologischen Ebene die Vermischung von nationalistischen und sozialistischen Elementen und im or-

35 Vgl. Laqueur, Faschismus (Anm. 3), S. 226-234.

36 Vgl. Birsl/Bucak/Zeyrek, Religiöser Fundamentalismus oder politischer Rechtsextremisms? (Anm. 4), S. 727.

37 Vgl. Richard Saage, Faschismus. Konzeptionen und historische Kontexte. Eine Einführung, Wiesbaden 2007.

38 Vgl. Ian Kershaw, Der NS-Staat. Geschichtsinterpretationen und Kontroversen im Überblick, Reinbek 1988,S. 89-124.

39 Vgl. Stanley Payne, Geschichte des Faschismus. Aufstieg und Fall einer europäischen Bewegung, Berlin 2001. 
ganisatorischen Bereich das Bestehen einer aktivierbaren Massenbewegung, beides im Sinne einer mentalen und politischen Homogenisierung der Gesellschaft. ${ }^{40}$

\section{2 Faschismus - Analyse und Kritik}

Hinsichtlich der historisch-politischen Komponente der Faschismus-Zuordnung an den Islamismus lässt sich in der Tat eine enge Kooperation zwischen Vertretern dieses politischen Lagers und dem deutschen Nationalsozialismus belegen. Insbesondere spielte dabei die Person des Mufti von Jerusalem Amin el-Husseini eine wichtige Rolle, warb er doch muslimische Freiwillige für die Waffen-SS an und forderte eine Verschärfung der Judenverfolgung. ${ }^{41}$ Aber auch darüber hinaus zeigten sich andere bedeutende Organisationen und Personen zu einer solchen Zusammenarbeit bereit. ${ }^{42}$ Dies muss vor dem Hintergrund gemeinsamer Feindbilder auf unterschiedlicher Grundlage in Gestalt der Briten als außenpolitische Gegner bzw. örtliche Kolonialmacht und der Juden als rassistisches Negativ-Bild bzw. zionistische Einwanderer gesehen werden. Politische Kooperation steht zwar für gewisse Gemeinsamkeiten, daraus lässt sich aber nicht zwingend eine inhaltliche Gleichsetzung der Phänomene ableiten.

Genau darin besteht aber das methodische Hauptproblem der Zuordnung des Islamismus zum Faschismus oder Rechtsextremismus: Bei beiden Begriffen handelt es sich um Bezeichnungen, die auf politische Bestrebungen mit einer besonderen ideologischen Ausrichtung verweisen. Genau dieser inhaltliche Gesichtspunkt bleibt konstitutiv für das Verständnis von »Faschismus « und »Rechtsextremismus«, während die Bezeichnungen »Extremismus «, »Fundamentalismus « und »Totalitarismus « primär auf formale Merkmale der Herrschaftstechnik oder Ideologie abstellen. So zutreffend der Verweis auf Gemeinsamkeiten wie die Ablehnung der Demokratie und Individualität, der Menschenrechte und des Westens ist, so kann daraus allenfalls eine Gemeinsamkeit in der Frontstellung gegen die damit verbundenen Normen und Werte postuliert werden. Eine solche lässt sich auch bei den kommunistischen Bewegungen und Systemen ausmachen, was jedoch nicht die Rede vom »Islam-Kommunismus « als sinnvoll erscheinen ließe.

Die Deutung des Islamismus als Bestandteil von Faschismus und Rechtsextremismus wäre nur sinnvoll, wenn er über entsprechende ideologische Grundprägungen verfügen würde. Gerade hier lassen sich aber fundamentale Differenzen ausmachen: Während für den Rechtsextremismus die ethnische Zugehörigkeit einen herausragenden Stellenwert einnimmt, spielt sie für den Islamismus überhaupt keine Rolle. Entscheidend für diese politische Bestrebung ist die Orientierung an einer besonderen Interpretation des Islam, wofür »Nation« oder »Rasse« als individuelle Merk-

40 Vgl. Robert O. Paxton, Anatomie des Faschismus, München 2006.

41 Vgl. Klaus Gensicke, Der Mufti von Jerusalem und die Nationalsozialisten. Eine politische Biographie Amin el-Husseinis, Darmstadt 2007.

42 Vgl. Klaus-Michael Mallmann/Martin Cüppers, Halbmond und Hakenkreuz. Das Dritte Reich, die Araber und Palästina, Darmstadt 2007. 
male der Gläubigen von keiner Bedeutung sind. Demgegenüber handelt es sich bei »Faschismus « und »Rechtsextremismus « trotz der mitunter auszumachenden Rückgriffe auf religiöse Bezugspunkte um säkulare Bewegungen, meist mit negativen Einstellungen gegenüber Menschen mit dem ethnischen Hintergrund der meisten Muslime. Dies dürfte auch eine systematische Kooperation von Islamisten und Rechtsextremisten in Zukunft verhindern. ${ }^{43}$

\section{Angemessenbeit der Fundamentalismus-Zuordnung}

\subsection{Fundamentalismus - Begründung und Definition}

Die häufigste Zuschreibung für den Islamismus erfolgte wohl mit dem Fundamentalismus-Begriff, setzt man doch mitunter beide Bezeichnungen sogar gleich. Auch die Rede vom »islamischen Fundamentalismus« macht dieses Verständnis deutlich. Es kam in der öffentlichen und wissenschaftlichen Wahrnehmung verstärkt nach der »Islamischen Revolution « im Iran 1979 auf und sollte eine besonders dogmatische und fanatische Einstellung im Sinne eines politischen Islam kennzeichnen. ${ }^{44}$ Dabei verwies man insbesondere auf die wortwörtliche Auslegung des Koran und die Idealisierung der Frühgeschichte des Islam. Gleichzeitig wies die Forschung darauf hin, dass es solche Auffassungen nicht nur in dieser Religion gab. ${ }^{45}$ Als Reaktion auf gesellschaftliche Modernisierung und Umbrüche bildeten sich auch in anderen Religionen derartige Tendenzen heraus, was zur Auffächerung des Begriffs in die Varianten eines christlichen, islamischen und jüdischen Fundamentalismus führte. ${ }^{46}$

Irritierend wirkte dieses Verständnis zunächst dadurch, dass der gewählte Terminus eigentlich aus der Geschichte des US-amerikanischen Protestantismus stammt. ${ }^{47}$ Es handelte sich ursprünglich um die Selbstbezeichnung einer Tendenz innerhalb dieser Glaubensrichtung, die erstmals in den 1910er Jahre aufkam und starken gesellschaftlichen Einfluss erlangte. Die aus Bibelkonferenzen entstandene Bewegung erhob die Forderung nach einer Rückkehr zu den Fundamenten und formulierte eine heftige Kritik an modernen Tendenzen im christlichen Glauben. Sie trat darüber hinaus für die absolute Irrtumslosigkeit der Heiligen Schrift und gegen die Evolutionstheorie über die Entstehung des Menschen ein. Da sich in diesem letztge-

43 Vgl. Armin Pfahl-Traughber, Das Verhältnis von Islamisten und Rechtsextremisten. Droht eine gemeinsame Front über den Antisemitismus, in: www.bpb.de/themen/ YN4KP6.html.

44 Vgl. Bruno Funk, Der geschundene Pfau. Der Iran unter den Ayatollahs, Hamburg 1985.

45 Vgl..Gilles Kepel, Die Rache Gottes. Radaikale Moslems, Christen und Juden auf dem Vormarsch, München 1991.

46 Vgl. Thomas Meyer, Fundamentalismus in der modernen Welt. Die Internationale der Unvernunft, Frankfurt/M. 1989.

47 Vgl. Martin Riesebrodt, Fundamentalismus als patriarchalische Protestbewegung. Amerikanische Protestanten (1910-28) und iranische Schiiten (1961-79), im Vergleich, Tübingen 1990. 
nannten Aspekt auch formale Gemeinsamkeiten mit den seit Anfang der 1970er Jahre auszumachenden Entwicklungen in anderen Religionen ergaben, konnte die Bezeichnung auch sinnvoll auf derartige Tendenzen als allgemeinen Typus-Begriff übertragen werden..$^{48}$

Darüber hinaus entstand noch ein Fundamentalismus-Verständnis, das auch auf Entwicklungen im säkularen Bereich in Gestalt von politischen und sozialen Phänomenen Bezug nahm. Teilweise wurde dabei der Terminus als politisches Schlagwort, teilweise als wissenschaftliche Kategorie gebraucht. ${ }^{49}$ In letztgenanntem Sinne bezeichnet »Fundamentalismus « Einstellungen und Verhaltensweisen, welche Merkmale wie absoluter Wahrheitsanspruch und elitärer Erkenntnisanspruch, geschlossene Denksysteme und dualistisches Weltbild aufwiesen. Die eigenen Auffassungen gelten in dieser Sicht als die einzig richtigen Betrachtungen zu Gesellschaft, Politik oder Religion, sie müssen sich daher weder einer empirischen Überprüfung noch einer kritischen Reflexion aussetzen lassen. ${ }^{50}$ Als solche Fundamente stehen die jeweiligen Inhalte dann gegen als feindlich anzusehende Werte aus anderen Bereichen. Damit verbindet sich die Ablehnung von gesellschaftlicher Differenz und sozialem Pluralismus. ${ }^{51}$

\subsection{Fundamentalismus - Analyse und Kritik}

Allgemein kann schon nach der Betrachtung der unterschiedlichen Begriffsverständnisse von »Fundamentalismus « gesagt werden, dass sich dieser Terminus nahe$\mathrm{zu}$ in allen Fällen inhaltlich angemessen auf den Islamismus anwenden lässt. Dies dürfte am einfachsten für das gegen Ende der vorherigen Ausführungen vorgetragene allgemeine Verständnis nachvollziehbar sein, stellt dieses doch auf die Eigenschaften des Absolutheitsanspruchs und Dogmatismus, der Feindbildorientierung und Intoleranz ab. Da diese Einstellungen den Islamisten schon in nahezu idealtypischer Weise eigen sind, kann eine solche Zuordnung ohne inhaltliche Probleme vorgenommen werden. Der hier zum Ausdruck kommende Fundamentalismus sieht allein im Islam die Lösung für alle gesellschaftlichen Probleme, wobei aber meist noch nicht einmal ansatzweise ein angestrebtes Idealmodell inhaltlich skizziert wird. Hinzu kommt die Ablehnung von allen anders lautenden Islam-Deutungen und der Anspruch auf Alleinvertretung.

Beschränkt man das Fundamentalismus-Verständnis mehr auf die Entwicklungen innerhalb der Religionen ${ }^{52}$, kann auch hier der Islamismus unter dieser Bezeichnung subsummiert werden. Gleichwohl gilt es zu bedenken, dass der Islamismus auch als islamischer Fundamentalismus gelten kann, aber nicht jeder islamische Fundamen-

48 Vgl. Martin E. Marty/R. Scott Appleby, Herausforderung Fundamentalismus. Radikale Christen, Moslems und Juden im Kampf gegen die Moderne, Frankfurt/M. 1996.

49 Vgl. Stephan H. Pfürtner, Fundamentalismus. Die Flucht ins Radikale, Freiburg 1991.

50 Vgl. Kurt Salamun (Hrsg.), Fundamentalismus »interdisziplinär«, Wien 2005.

51 Vgl. Thomas Meyer, Fundamentalismus. Aufstand gegen die Moderne, Reinbek 1989.

52 Vgl. Wilfried Röhrich, Die Macht der Religionen. Glaubenskonflikte in der Weltpolitik, München 2004. 
talist ein Islamist im oben definierten Sinne sein muss. Eine dogmatische Auslegung des Koran wäre sicherlich als fundamentalistisch zu bezeichnen, sie wäre allerdings erst dann islamistisch, wenn damit ein politischer Herrschaftsanspruch einher geht. Dies dürfte angesichts der engen Verkopplung von Gesellschaft, Politik und Religion im Islam häufig der Fall sein, es muss aber nicht notwendigerweise immer eine solche Dimension der Glaubensinterpretation vorherrschen. Insofern bildet die Bezeichnung »islamischer Fundamentalismus ebenfalls eine Sammelbezeichnung, worunter sowohl islamistische wie nicht-islamistische Interpretation dieser Religon zu fassen wären.

Dies nötigt daher zu einer Unterscheidung von islamistischen, also stark politisch ausgerichteten und orthodoxen, also mehr religiös orientierten Auffassungen vom Islam. ${ }^{53}$ Beide Richtung zeigen sich geprägt von Absolutheitsansprüchen und Ausgrenzungstendenzen, welche allerdings vielen Religionen mehr oder minder stark eigen sind. ${ }^{54}$ So lange sie sich nur auf die Ebene des Glaubens beziehen, stellen sie kein bedeutendes Problem für demokratische Verfassungsstaaten und offene Gesellschaften dar. ${ }^{55}$ Beabsichtigen sie aber ihre religiösen Dogmen und Gültigkeitsansprüche im Sinne einer politischen Bestrebung auf die gesamte Gesellschaft zu übertragen, so führt dies notwendigerweise zu einer Aufhebung von Demokratie, Menschenrechten und Pluralismus. Insofern können auch nicht alle Formen wie kultureller oder religiöser Art, aber sehr wohl die politischen Varianten des Fundamentalismus unter die oben definierte Auffassung von politischem Extremismus subsummiert werden. ${ }^{56}$

\section{Angemessenbeit der Totalitarismus-Zuordnung}

\subsection{Totalitarismus - Begründung und Definition}

Und schließlich gilt es noch die Deutung des Islamismus als Totalitarismus zu thematisieren: Hier lassen sich solche Etikettierungen ohne nähere Begründung selbst in Monographien mit einschlägigen Titeln ausmachen. Darin verweist man lediglich auf die Gefahren für Demokratie und Freiheit und sieht das Aktuelle des Phänomens in nicht-staatlichen Akteuren als Träger des »neuen Totalitarismus «. ${ }^{77}$ In anderen Fällen

53 Vgl. Michael Lüders, Allahs langer Schatten. Warum wir keine Angst vor dem Islam haben müssen, Freiburg 2007, S. 112 mit allerdings anderem Begriffsverständnis.

54 Vgl. Armin Pfahl-Traughber, Der fundamentalistische Charakter von Religionen und die Grenzen der Religionsfreiheit im säkularen Rechtsstaat. Eine demokratietheoretisch und ideologiekritisch ausgerichtete Erörterung anhand von Christentum und Islam, in: Eric Hilgendorf (Hrsg.), Wissenschaft, Religion und Recht. Hans Albert zum 85. Geburtstag, Berlin 2006, S. 177-199.

55 Vgl. Rolf Poscher, Du musst nicht verfassungstreu sein, in: Frankfurter Allgemeine Zeitung vom 28. Juni 2007, S. 7.

56 Vgl. Armin Pfahl-Traughber, Extremismus als politische Variante des Fundamentalismus. Gemeinsamkeiten und Unterschiede bei der Ablehnung der kulturellen Moderne, in: Mut, Nr. 334/Juni 1995, S. 58-65.

57 Vgl. Tibi, Der neue Totalitarismus (Anm. 8), S. 19-21. 
stellen Anhänger dieser Auffassung auf die vom Islamismus ebenso wie vom Kommunismus und Nationalsozialismus beabsichtigte grundlegende Veränderung der ganzen Welt im Namen einer religiös erscheinenden Offenbarung ab. Dies führe zur Ersetzung des Staates durch eine technischen Einrichtung, die von einer Elite wie Parteifunktionären oder Religionsgelehrten beherrscht werde..$^{58}$ Oder man verweist auf gemeinsame Strukturmerkmale wie die ideologische Durchdringung aller Lebensbereiche, den Zwang zum öffentlichen Bekenntnis oder die Indoktrinierung von Kindern. ${ }^{59}$

Die Bezeichnungen »totalitär« bzw. »Totalitarismus « entstammten allerdings einem ganz anderen politischen Kontext: Erstmals nutzten den erstgenannten Terminus Anfang der 1920er Jahre Gegner des Mussolini-Regimes, um dessen Streben nach einem totalen Staat zu kritisieren. Später übernahm der faschistische Diktator selbst diese Bezeichnung und bekannte sich affirmativ zu der damit verbunden Absicht. Ende der 1920er Jahre stellte eine Reihe von Intellektuellen, Politikern und Wissenschaftlern vergleichende Betrachtungen zum bolschewistischen System in der Sowjetunion an. ${ }^{60}$ Aus diesen Überlegungen entstanden ab den 1930er Jahren Analysen, die sich allerdings überwiegend auf einen Vergleich der Herrschafsstrukturen des Nationalsozialismus und Stalinismus bezogen. Einschlägige Forschungen mündeten nach 1945 in eigenständigen Totalitarismustheorien, die einen neuen Typus politischer Herrschaft beschreiben wollten. ${ }^{61}$

Am einflussreichsten wirkten zwei Ansätze aus den 1950er Jahren: Die Philosophin Hannah Arendt sah in der Herausbildung geschlossener Ideologien und in der Permanenz des Terrors das Wesen des Totalitarismus. ${ }^{62}$ Und der Politologe Carl J. Friedrich stellte einen Merkmalskatalog mit sechs Wesenszügen für solche politischen Systeme auf: eine geschlossene Ideologie, eine Staatspartei, eine terroristische Geheimpolizei, ein Nachrichtenmonopol des Staates, ein Waffenmonopol und eine zentral gelenkte Wirtschaft. ${ }^{63}$ Nicht alle Eigenschaften konnten durch die spätere Faschismus- und Kommunismusforschung bestätigt werden. ${ }^{64}$ Diese Einsicht bewog zu einer kritischen Revision und inhaltlichen Weiterentwicklung des Totalitarismus-Verständnisses. ${ }^{65}$ Der Politologe Juan J. Linz benannte etwa Mitte der

58 Vgl. Bauer, Der dritte Totalitarisms (Anm. 7).

59 Vgl. Friedbert Pflüger, Ein neuer Weltkrieg? Die islamistische Herausforderung des Westens, München 2004, S. 11 und 74.

$60 \mathrm{Vgl}$. Jens Petersen, Die Entstehung des Totalitarismusbegriffs in Italien, in: Manfred Funke (Hrsg.), Totalitarismus. Ein Studien-Reader zur Herrschaftsanalyse moderner Diktaturen, Düsseldorf 1978, S. 105-128.

61 Vgl. Bruno Seidel/Siegfried Jenkner (Hrsg.), Wege der Totalitarismus-Forschung, Darmstadt 1968.

62 Vgl. Hannah Arendt, Elemente und Ursprünge totaler Herrschaft (1951), München 1986.

63 Vgl. Carl J. Friedrich, Totalitäre Diktatur. Unter Mitarbeit von Zbigniew K. Brzezinski, Stuttgart 1957.

64 Vgl. Armin Pfahl-Traughber, Klassische Totalitarismuskonzepte auf dem Prüfstand. Darstellung und Kritik der Ansätze von Arendt, Friedrich, Popper und Voegelin, in: Uwe Backes/Eckhard Jesse (Hrsg.), Jahrbuch Extremismus \& Demokratie. Bd. 16, Baden-Baden 2004, S. 31-58.

65 Vgl. Eckhard Jesse (Hrsg.), Totalitarismus im 20. Jahrhundert. Eine Bilanz der Forschung, Baden-Baden 1996. 
1970er Jahre folgende Charakteristika totalitärer Systeme: ein monistisches, aber nicht monolitisches Machtzentrum, eine autonome und exklusive Ideologie und die aktive Beteiligung und Mobilisierung der Massen. ${ }^{66}$

\subsection{Analyse und Kritik}

Ein Problem für die Anwendung des Totalitarismus-Begriffs auf den Islamismus ergibt sich bereits dadurch, dass das damit verbundene Konzept nicht auf die Bewegungs-, sondern die Systemebene bezogen ist. In erster Linie sollte es um einen Diktaturvergleich gehen. Bislang konnten allerdings nur drei theokratische Diktaturen islamistischer Ausrichtung in den letzten beiden Jahrzehnten des 20. Jahrhunderts ausgemacht werden: die Regime in Afghanistan, im Iran und im Sudan ${ }^{67}$, wobei erstere und letztere nur relativ kurze Zeit bestanden und es sich im Sudan nur um eine partielle Herrschaft handelte. Für die Einschätzung der Taliban-Herrschaft in Afghanistan als totalitäres System spricht der rigorose Anspruch, die Gesellschaft vollständig nach ihren Maßstäben zu kontrollieren. Dies zeigte sich insbesondere anhand der Vorgaben für Frauen in Gestalt von Arbeitsverboten, Kleidungsvorschriften und Schulverbot. Indessen nutzte man kaum moderne Technik zur Herrschaftsabsicherung und -legitimation. ${ }^{68}$

Für das islamistische Regime im Iran lässt sich für das erste Jahrzehnt seiner Existenz unter Führung Khomeinis eine totalitäre Dimension konstatieren ${ }^{69}$ : Die »Regentschaft des Rechtsgelehrten « erfolgte mit allen Machtmitteln und ohne Kontrollmöglichkeiten. Khomeini stand somit über Exekutive, Judikative und Legislative und jede Kritik an ihm musste als Verwerfung von Gottes Willen gelten. Rigoros ging man gegen Andersdenkende und Oppositionelle vor: Alle nicht-islamischen Organisationen und Partien wurden verboten, die neue Geheimpolizei und die »Revolutionswächter « erstickten brutal alle kritischen Regungen. Nach dem Tod Khomeinis 1989 ging die Konzentration der Macht auf eine Person zurück, die Geschlossenheit der Führung löste sich ansatzweise auf. Als Reaktion auf die unterschiedlichen innen- wie außenpolitischen Umbruchprozesse entstand gegenüber dem dogmatisch ausgerichteten ein reformerisch orientierter Flügel. Es handelt sich allerdings nur um zwei verschiedene Vertreter des theokratischen Systems. ${ }^{70}$

66 Vgl. Juan J. Linz, Totalitäre und autoritäre Regime (1975), Berlin 2000, S. 25 und 64.

67 Vgl. Armin Pfahl-Traughber, Staatsformen im 20. Jahrhundert I: Diktatorische Systeme, in: Alexander Gallus/Eckhard Jesse (Hrsg.), Staatsformen. Modelle politischer Ordnung von der Antike bis zur Gegenwart. Ein Handbuch, Köln 2004, S. 223- 280, hier S. 261-267.

68 Vgl. Ahmed Rashid, Taliban. Afghanistans Gotteskrieger und der Dschihad, München 2001.

69 Vgl. Wolfgang-Uwe Friedrich, Formen des Totalitarismus. Zur Phänomenologie ideologischer Herrschaft im 20. Jahrhundert, in: Eckhard Jesse/Steffen Kailitz (Hrsg.), Prägekräfte des 20. Jahrhunderts. Demokratie, Extremismus, Totalitarismus, München 1997, S. 251-283, hier S. 279-281.

70 Vgl. Wahied Wahdat-Hagh, »Die Islamische Republik Iran«. Die Herrschaft des politischen Islam als eine Spieart des Totalitarismus, Münster 2003. 
In welchem Maße lässt sich nun der Totalitarismus-Begriff auf den Islamismus in seiner Bewegungsform anwenden? Dies kann vor dem Hintergrund seines ursprünglichen Bezuges auf die Diktaturform lediglich auf den beabsichtigten Herrschaftsanspruch bzw. hinsichtlich der dabei artikulierten Ideologie möglich sein. Betrachtet man die Idee und Zielsetzung des "Gottesstaates « im islamistischen Sinne näher, so lassen sich diese durchaus als Form des totalitären Denkens verstehen. ${ }^{71}$ Den Vertretern dieser Ideologie geht es um die möglichst vollständige Durchdringung und Kontrolle der Gesellschaft in ihrem ideologischen Sinne, wobei auch der private Bereich durch Vorgabe für ein bestimmtes Frauenbild oder spezifische Kleidungsvorschriften erfasst werden würde. Darüber hinaus beabsichtigt man eine gezielte Indoktrinierung und Mobilisierung der Gesellschaft über die religiöse Erziehung und Unterweisung, was ebenfalls auf eine Abschaffung des Pluralismus und Gleichschaltung der Gesellschaft im totalitären Sinne hinaus liefe.

\section{Schlusswort}

Bilanziert man die vorstehenden Ausführungen, so lässt sich folgendes sagen: Der Islamismus wird in der öffentlichen und wissenschaftlichen Diskussion häufig mit den Begriffen »Extremismus «, »Faschismus «, »Fundamentalismus « und »Totalitarismus « belegt. Dabei dient nicht nur den meisten Politikern, sondern auch vielen Wissenschaftlern eine derartige Ettikettierung lediglich als pauschale Zuordnung, mangelt es doch meist an einer inhaltlichen Begründung für die getroffene Einschätzung. Konfrontiert man nun das Phänomen des Islamismus mit dem wissenschaftlichen Verständnis der vorgenannten Begriffe, so ergibt sich aber durchaus eine inhaltliche Angemessenheit, allerdings nur für die auf formale Merkmale ausgerichteten Bezeichnungen »Extremismus«, »Fundamentalismus« und »Totalitarismus«. Sie beziehen sich auf eine Frontstellung gegen die Grundwerte einer modernen Demokratie, die Absolutsetzung und Dogmatisierung der eigenen Positionen oder die Absicht einer umfassenden Kontrolle der Gesellschaft.

Derartige Ausrichtungen von Handlungsweisen oder Strukturen von Ideologien können verschiedene Inhalte annehmen, was es auch erlaubt den Islamismus als Subphänomen oder Teilbereich der erwähnten Sammelbezeichnungen zu deuten. Im Unterschied zu diesen handelt es sich bei den Bezeichnungen »Faschismus «und »Rechtsextremismus « um Begriffe, die über eine bestimmte inhaltliche Komponente der Ideologie definiert werden. Hierbei spielt insbesondere die Überbewertung ethnischer Zugehörigkeit, wie sie sich in Nationalismus und Rassismus artikuliert, eine bedeutende Rolle. Gerade diese Auffassung kann den Islamisten aber nicht zugeordnet werden. Formale Gemeinsamkeiten, identische Feindbilder oder partielle Kooperation lassen zwar Ähnlichkeiten erkennen, erlauben aber keine inhaltliche Gleichsetzung. Insofern können Bezeichnungen wie »Islamo-Faschismus « als politische Schlagworte gelten, welche aus wissenschaftlicher Sicht unangemessen sind.

71 Vgl. Pfahl-Traughber, Islamismus als extremistisches und totalitäres Denken (Anm. 32). 
Wie steht es nun aber um die Erklärungskraft der vorgenommenen Zuordnungen des Islamismus zum Extremismus, Fundamentalismus oder Totalitarismus? Hier muss schlicht konstatiert werden, dass diese Begriffe primär zur Kategorisierung des Phänomens dienen und dabei auf das Spannungverhältnis zu moderner Demokratie und offener Gesellschaft abgestellt wird. Damit lässt sich nicht das Aufkommen und die Entwicklung des Islamismus als politische Bestrebung oder terroristische Handlungsweise erklären. Allenfalls stellen die Zuordnungen auf den Charakter einer Gegenbewegung zu einer von Differenz und Entwicklung, Offenheit und Pluralität geprägten Gesellschaftsordnung ab. Eine ausführlichere Analyse von Bedingungsfaktoren und Ursachen kann man damit nicht vornehmen. Gleichwohl bedarf es solcher Zuordnungen des Islamismus, um sich der damit zusammenhängenden Gefahren für das Bestehen von Demokratie und Menschenrechten zu vergegenwärtigen.

\section{Zusammenfassung}

Der Islamismus wird in der öffentlichen und wissenschaftlichen Diskussion häufig mit den Begriffen »Extremismus«, »Faschismus«, »Fundamentalismus« und »Totalitarismus « belegt. Dabei dient nicht nur den meisten Politikern, sondern auch vielen Wissenschaftlern eine derartige Ettikettierung lediglich als pauschale Zuordnung, mangelt es doch meist an einer inhaltlichen Begründung für die getroffene Einschätzung. Konfrontiert man nun das Phänomen des Islamismus mit dem wissenschaftlichen Verständnis der vorgenannten Begriffe, so ergibt sich aber durchaus eine inhaltliche Angemessenheit, allerdings nur für die auf formale Merkmale ausgerichteten Bezeichnungen »Extremismus «, »Fundamentalismus « und »Totalitarismus «. Sie beziehen sich auf eine Frontstellung gegen die Grundwerte einer modernen Demokratie, die Absolutsetzung und Dogmatisierung der eigenen Positionen oder die Absicht einer umfassenden Kontrolle der Gesellschaft. Derartige Ausrichtungen von Handlungsweisen oder Strukturen von Ideologien können verschiedene Inhalte annehmen, was es auch erlaubt den Islamismus als Subphänomen oder Teilbereich der erwähnten Sammelbezeichnungen zu deuten. Anders verhält es sich bei den Bezeichnungen »Faschismus « und »Rechtsextremismus «, die über eine bestimmte inhaltliche Komponente der Ideologie - insbesondere die Überbewertung ethnischer Zugehörigkeit wie Nationalismus und Rassismus - definiert werden. Gerade diese Auffassungen kann den Islamisten aber nicht zugeordnet werden. Insofern stellen Bezeichnungen wie Islamo-Faschismus « politische Schlagworte dar, welche aus wissenschaftlicher Sicht unangemessen sind.

\section{Summary}

In public and scientific debate Islamism is frequently associated with the expressions »extremism «, »fascism«, »fundamentalism« and »totalitarianism «. This kind of labelling serves not only the majority of politicians, but also many scientists, as 
merely overall classification which is generally lacking substance to support it. Confronting the phenomenon of Islamism with the scientific understanding of the above terms is however only reasonable if the formal characteristics of the definitions of »extremism «, »fundamentalism « and »totalitarianism « are used. These are premised against the basic values of a modern democracy, the absolute dogma of one's position or the aim of exerting total control over a society. The orientation of behaviour and structure of ideologies may take various forms which allows Islamism to be classified as a sub-phenomenon or element of the above collective definitions. The terms »fascism « and »right wing extremism « however differ. They are defined by specific ideologies, in particular the over emphasis on ethnic identity in terms of nationalism and racism. Islamists cannot be defined within this concept. In this respect expressions such as »islamo-fascism « are political headlines which, from a scientific perspective, are not appropriate.

Armin Pfabl-Tranghber, Islamism - The new extremism, fascism, fundamentalism and totalitarianism? A discussion on the appropriateness and the strength of this argument

\section{Bewertung der DDR-Vergangenheit}

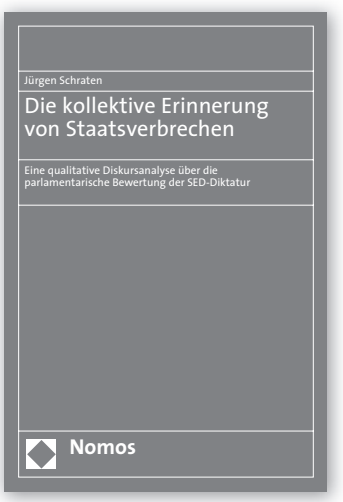

\section{Die kollektive Erinnerung von Staatsverbrechen}

Eine qualitative Diskursanalyse über die parlamentarische Bewertung der SED-Diktatur Von Jürgen Schraten 2008, 182 S., brosch., 34,-€, ISBN 978-3-8329-3164-3

Die qualitative Diskursanalyse fördert den demokratischen Kern der Debatten des Deutschen Bundestages zur Bewertung der DDR-Vergangenheit zutage. Der Autor formt aus dem wortreichen Streit über Ursachen und Folgen der Diktatur einen beobachtbaren Diskurs, aus dem ein antitotalitäres Fundament der gesamtdeutschen Bundesrepublik hervorgeht.

Bitte bestellen Sie bei Ihrer Buchhandlung oder bei Nomos | Telefon 07221/2104-37 | Fax-43 | www.nomos.de|sabine.horn@nomos.de

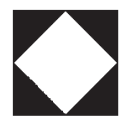

Nomos 